\title{
Claudin 18.2 expression in esophageal adenocarcinoma and its potential impact on future treatment strategies
}

\author{
VALESKA MOENTENICH $^{1 *}$, FLORIAN GEBAUER ${ }^{2 *}$, ERDEM COMUT $^{3}$, ARMIN TUCHSCHERER $^{1}$, \\ CHRISTIANE BRUNS ${ }^{2}$, WOLFGANG SCHROEDER ${ }^{2}$, REINHARD BUETTNER ${ }^{4}$, HAKAN ALAKUS ${ }^{2}$, \\ HEIKE LOESER $^{4}$, THOMAS ZANDER ${ }^{1}$ and ALEXANDER QUAAS ${ }^{4}$
}

\begin{abstract}
Departments of ${ }^{1}$ Oncology and Hematology and ${ }^{2}$ Visceral Surgery, University of Cologne, D-50937 Cologne, Germany; ${ }^{3}$ Institute of Pathology, Pammukale University, Denizli 20160, Turkey; ${ }^{4}$ Institute of Pathology, University of Cologne, D-50937 Cologne, Germany
\end{abstract}

Received May 25, 2019; Accepted November 12, 2019

DOI: 10.3892/ol.2020.11520

\begin{abstract}
The incidence of esophageal adenocarcinoma (EAC) has rapidly increased, particularly in the Western world. Despite improvements in perioperative treatments, the overall survival of patients remains low. Claudin 18.2 is a tight junction protein that is exclusively expressed in the gastric epithelia. However, following malignant transformation, gastric cancer metastases maintain this expression. Therefore, claudin 18.2 is a promising target for immunotherapy. Previous clinical trials have revealed improved anti-tumor activity in patients treated with an anti-claudin antibody by investigating the expression of claudin 18.2 in tumor cells. However, there is currently very limited data on the importance of claudin 18.2 expression in EAC. The present study analyzed the distribution of claudin 18.2 using immunohistochemistry in 485 patients with EAC, including their lymph node metastases. Additionally, these results were associated with clinical and molecular data. Claudin 18.2 was detected in $89 / 485$ patients (18.4\%). No correlations between expression and clinicopathological data (sex, age, pT stage, lymph node metastasis and grading) were observed. However, significantly decreased claudin 18.2 expression was observed in tumor types with upregulated human epidermal growth factor receptor 2 expression $(\mathrm{P}=0.036)$. Additionally, neoadjuvant treatment did not have any significant impact on claudin 18.2 expression $(\mathrm{P}=0.331)$. To the best of our knowledge, the present study is the largest systematic investigation of claudin 18.2 protein expression in EAC. The results obtained suggested that claudin 18.2 may
\end{abstract}

Correspondence to: Mrs. Valeska Moentenich, Department of Oncology and Hematology, University of Cologne, 62 Kerpener Strasse, D-50937 Cologne, Germany

E-mail: valeska.moentenich@uk-koeln.de

*Contributed equally

Key words: esophageal adenocarcinoma, claudin 18.2 expression, immunotherapy, targeted therapy, IMAB362 serve as a promising therapeutic target in a substantial number of patients with EAC.

\section{Introduction}

Esophageal cancer (including squamous cell carcinoma and adenocarcinoma) is the eighth most frequently diagnosed cancer globally (1). The incidence of adenocarcinoma has rapidly increased in the Western world in recent decades $(2,3)$. In the United States of America, the incidence increased from 3.6 per million in 1973 to 25.6 per million in 2006 (4). The substantial increase in esophageal adenocarcinoma (EAC) is presumably caused by a higher incidence of known risk factors, including obesity and gastroesophageal reflux (5). EAC is thought to arise from Barrett's metaplasia, caused by chronic gastroesophageal reflux. The accumulation of various mutations, copy-number variations and chromothripsis causing genetic instability finally result in carcinogenesis (6-8). Despite improvements in perioperative treatments, the overall survival (OS) time of patients with esophageal carcinomas remains low. The relative 5 year survival rate remains poor, at $21 \%$ in both sexes (20.2 and $22.8 \%$ for adenocarcinomas and squamous cell carcinomas, respectively) $(9,10)$.

Claudins are proteins required for the formation of tight junctions and paracellular barriers (11-13). At least 27 different claudins have been discovered in humans so far (14). Claudins have four transmembrane domains and a molecular mass ranging between 20 and $27 \mathrm{kDa}$ (14). In 2008, Sahin et al (15) identified isoform 2 of claudin 18 (claudin 18.2) as a highly selective marker for differentiated gastric mucosa epithelial cells. Claudin 18.2 expression was revealed to occur exclusively in normal gastric tissue and was not detected in any other examined tissue. In addition, the study demonstrated a significantly higher expression of claudin 18.2 in gastric adenocarcinomas and their metastases, including carcinomas of the pancreas, esophagus, ovaries and lungs. Additionally, a correlation between the histological subtype of carcinomas and expression of claudin 18.2 was revealed. EACs, mucinous ovarian carcinomas and ductal adenocarcinomas of the pancreas were positive for claudin 18.2 expression. By contrast, no expression was detected in squamous cell carcinomas of the esophagus, serous ovarian 
carcinomas and islet cell carcinomas of the pancreas $(15,16)$. As claudin 18.2 exhibits distinct expression patterns and is located in the outer cell membrane, it is an attractive target for targeted therapies $(15,16)$. To the best of our knowledge, the expression status of claudin 18.2 in EAC has only been investigated in one previous study, in which Sahin et al (15) demonstrated the expression of claudin 18.2 in 17/22 EAC tissues (78\%) using an in-house developed mouse monoclonal antibody.

IMAB362 (claudiximab or zolbetuximab) is a novel chimeric immunoglobulin G1 antibody, developed by Ganymed Pharmaceuticals, which is able to specifically bind to claudin 18.2 on the cell surface $(16,17)$. Subsequent to binding to claudin 18.2, IMAB362 stimulates cellular and soluble immune effectors that activate antibody- and complement-dependent cytotoxicity, induce apoptosis and inhibit cell proliferation (16). Additionally, in combination with chemotherapy, IMAB362 enhances T-cell infiltration and induces the release of pro-inflammatory cytokines (16). IMAB362 is currently undergoing a number of phase I and IIb trials, which have revealed that IMAB362 is well-tolerated, exhibits anti-tumor activity and improves the disease control rate and progression-free survival time when administered as a monotherapy or in combination with chemotherapy. The most frequently observed treatment-associated adverse events include nausea and vomiting (18-21).

The aim of the present study was to detect the levels of claudin 18.2 expression in a large number of EAC samples, in addition to investigating its expression in metastases. Furthermore, these results were associated with clinicopathological and molecular data.

\section{Materials and methods}

Patients and tumor samples. Formalin-fixed and paraffinembedded primary tumor tissue microarrays (TMA) obtained from 685 patients with EAC that underwent primary surgical resection or resection following neoadjuvant therapy between January 1999 and November 2012 at the Department of General, Visceral and Cancer Surgery, University of Cologne (Cologne, Germany) were analyzed in the present study. Additionally, samples from 287 corresponding regional lymph node metastases were available. The patients underwent laparotomic or laparoscopic gastrolysis and right transthoracic en bloc esophagectomy with two-field lymphadenectomy of the mediastinal and abdominal lymph nodes. Reconstruction was performed by high intrathoracic esophagogastrostomy as previously described (22). Patients with advanced esophageal cancer (cT3, cNx and M0) received preoperative chemoradiotherapy [according to the ChemoRadiotherapy for Oesophageal Cancer Followed by Surgery Study protocol $(23,24)]$ or chemotherapy alone [according to the 5-fluorouracil, leucovorin, oxaliplatin and docetaxel protocol (24)]. Follow-up data were available for all patients. Patient characteristics are presented in Table I. Both Union for International Cancer and pTNM classifications were used for tumor staging $(25,26)$. Depending on the efficacy of neoadjuvant chemotherapy or radiochemotherapy, minor responders were defined as exhibiting a histopathological residual tumor of $\geq 10 \%$ (27).

The present study was ethically approved by the University of Cologne Ethics Committee (reference no. 13-091) and written informed consent was obtained from all patients. The procedures followed were in accordance with the Declaration of Helsinki.

Immunohistochemistry. TMA construction was performed as previously described $(28,29)$. In brief, tissue cylinders with a diameter of $1.2 \mathrm{~mm}$ were punched from selected tumor tissue blocks using an in-house developed semi-automated precision instrument and embedded in empty recipient paraffin blocks ( $4 \%$ formalin for $24 \mathrm{~h}$ at room temperature). Paraffin blocks were cut into $4 \mu \mathrm{m}$-thick sections, which were transferred onto an adhesive coated slide system. Freshly cut TMA sections were immunostained in one day and in one experiment. Slides were deparaffinized using standard protocols with Dewax (Leica Microsystems, Inc.) and 100\% ethanol, denaturated and exposed to heat-induced antigen retrieval for $5 \mathrm{~min}$ in an autoclave at $121^{\circ} \mathrm{C}$ and $\mathrm{pH} 9$ (Tris-EDTA-buffer). The TMA slides were incubated with a primary rabbit recombinant monoclonal antibody specific for claudin 18.2 (clone EPR 19202; 1:200; Abcam; cat. no. ab222512) using a Leica Bond Max automated system (35578; Leica Microsystems $\mathrm{GmbH}$ ) for $20 \min 37^{\circ} \mathrm{C}$. As a secondary antibody, the Bond polymer refine detection kit (Leica Microsystems $\mathrm{GmbH}$; cat. no DS9800) was used for $5 \mathrm{~min}$ at $37^{\circ} \mathrm{C}$. The primary antibody detects the same isoform 2 of Claudin 18 as described in clinical studies previously $(19,30)$. Normal gastric mucosa served as an internal control. The claudin 18.2 staining intensity was scored by two pathologists (AQ and HL) using a two-tier scoring system. The staining was described as follows: i) Negative, no claudin 18.2 expression; ii) low-grade expression, claudin 18.2 expression of any intensity in $5-49 \%$ of tumor cells; and iii) high-grade expression, claudin 18.2 expression of any intensity in $>49 \%$ of tumor cells (Fig. 1).

Statistical analysis. For the statistical analyses, a long follow-up period of the patients was available. Follow-up times ranged from a minimum of 48 months to 204 months. The follow-up of all patients was performed according to a standardized protocol. During the first two years, the clinical follow-up of patients was performed in the hospital every three months. Subsequently, annual examinations were performed. Follow-up examinations included obtaining a detailed history, clinical evaluation, abdominal ultrasound, chest X-ray and additional diagnostic procedures as required. Follow-up data were available for all patients. SPSS Statistics for Mac (Version 21; IMB Corp., Armonk, NY, USA) was used for statistical analysis. Data were presented as the means \pm standard deviation or median with range (min-max). Immunohistochemistry data were displayed as categorial variables $(0,1$ and 2$)$. All data were collected prospectively according to a standardized clinical protocol. Interdependence was calculated using a $\chi^{2}$ and Fisher's exact tests and displayed using cross-tables. Survival curves were plotted using the Kaplan-Meier method and analyzed using the log-rank test. Analyses were performed for independent prognostic factors of OS time, using the Cox regression model. All tests were two-sided. $\mathrm{P}<0.05$ was considered to indicate a statistically significant difference. 
Table I. Univariate analysis of clinical parameters. Sum of patients do not add to 485 patients due to missing clinical data or missing tumor spots on the tissue microarray.

Claudin 18.2 expression in esophageal adenocarcinoma

\begin{tabular}{|c|c|c|c|c|c|c|c|}
\hline \multirow[b]{2}{*}{ Clinical parameters } & \multicolumn{2}{|c|}{ Total } & \multicolumn{2}{|c|}{ Negative } & \multicolumn{2}{|c|}{ Positive } & \multirow[b]{2}{*}{ P-value } \\
\hline & $\mathrm{n}$ & $\%$ & $\mathrm{n}$ & $\%$ & $\mathrm{n}$ & $\%$ & \\
\hline Total number & 485 & 100 & 396 & 81.6 & 89 & 18.4 & \\
\hline Sex & & & & & & & 0.589 \\
\hline Female & 54 & 11.1 & 44 & 81.5 & 10 & 18.5 & \\
\hline Male & 431 & 88.9 & 352 & 81.7 & 79 & 18.3 & \\
\hline Age group & & & & & & & 0.448 \\
\hline$<65$ & 251 & 51.8 & 204 & 81.4 & 47 & 18.6 & \\
\hline$>65$ & 234 & 48.2 & 193 & 82.3 & 42 & 17.7 & \\
\hline pT stage & & & & & & & 0.289 \\
\hline 0 & 1 & 0.2 & 1 & 100 & 0 & 0.0 & \\
\hline 1 & 67 & 13.8 & 51 & 76.1 & 16 & 23.9 & \\
\hline 2 & 54 & 11.2 & 44 & 81.5 & 10 & 18.5 & \\
\hline 3 & 344 & 71.1 & 281 & 81.7 & 63 & 18.3 & \\
\hline 4 & 18 & 3.7 & 18 & 100 & 0 & 0.0 & \\
\hline pN stage & & & & & & & 0.434 \\
\hline 0 pos & 189 & 39.1 & 153 & 81.0 & 36 & 19.0 & \\
\hline 0 neg & 294 & 60.9 & 241 & 82.0 & 53 & 18.0 & \\
\hline UICC stage & & & & & & & 0.446 \\
\hline 1 & 99 & 20.5 & 76 & 76.8 & 23 & 23.2 & \\
\hline 2 & 111 & 23.0 & 94 & 84.7 & 17 & 15.3 & \\
\hline 3 & 201 & 41.7 & 163 & 81.1 & 38 & 18.9 & \\
\hline 4 & 71 & 14.7 & 60 & 84.5 & 11 & 15.5 & \\
\hline neoadj. & & & & & & & 0.331 \\
\hline No & 207 & 43.6 & 171 & 82.6 & 36 & 17.4 & \\
\hline Yes & 268 & 56.4 & 216 & 80.6 & 52 & 19.4 & \\
\hline Total & 475 & 100 & 387 & 81.5 & 88 & 18.5 & \\
\hline Her2 & & & & & & & 0.036 \\
\hline neg & 306 & 87.7 & 244 & 79.7 & 62 & 20.3 & \\
\hline pos & 43 & 12.3 & 40 & 93.0 & 3 & 7.0 & \\
\hline TP53 & & & & & & & 0.493 \\
\hline neg & 150 & 41.9 & 119 & 79.3 & 31 & 20.7 & \\
\hline pos & 208 & 58.1 & 172 & 82.7 & 36 & 17.3 & \\
\hline Total & 358 & 100 & 291 & 81.3 & 67 & 18.7 & \\
\hline ARID1A & & & & & & & 0.240 \\
\hline neg & 48 & 10.0 & 36 & 75.0 & 12 & 25.0 & \\
\hline pos & 432 & 90.0 & 355 & 82.2 & 77 & 17.8 & \\
\hline
\end{tabular}

UICC, Union for International Cancer Control; Her2, human epidermal growth factor receptor 2; TP53, tumor protein p53; ARID1A, AT-rich interaction domain $1 \mathrm{~A}$.

\section{Results}

Expression of claudin 18.2. The present study investigated 685 primary tumor types and 236 corresponding regional lymph node metastasis samples obtained from patients with EAC. However, a total of $485 / 685$ primary tumor type TMAs
(88.0\%) and 195/236 lymph node metastasis TMAs (82.7\%) were analyzed for claudin 18.2 expression. This was due to a lack of tissue or absence of unequivocal cancer tissue in certain TMAs. The median follow-up time for the entire cohort was 57.7 months with a calculated 5 year survival rate of $26.6 \%$. 


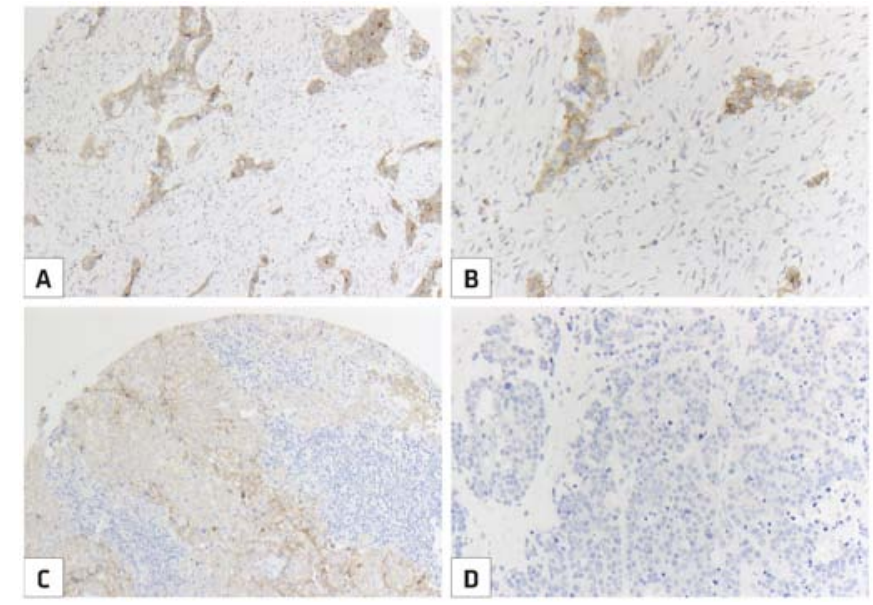

Figure 1. Immunohistochemistry results of claudin 18.2 in EAC. Claudin 18.2 positive EAC magnified by (A) x10 and (B) x20. (C) A claudin 18.2 positive lymph node metastasis (x10). (D) A claudin 18.2 negative EAC (x10). EAC, esophageal adenocarcinoma.

The expression of claudin 18.2 was observed in $89 / 485$ (18.4\%) primary tumor TMAs and in 35/195 (17.9\%) regional lymph node metastasis TMAs. There was no significant difference between the claudin 18.2 expression pattern in primary tumor types and corresponding lymph node metastases $(\mathrm{P}=1.000)$. No significant differences between claudin 18.2 expression and clinicopathological data (sex, age, pT stage, lymph node metastasis and grading) were observed (Table I). Additionally, no significant differences with tumor protein p53 (TP53) or AT-rich interaction domain 1A (ARID1A) mutations were identified. Furthermore, the administration of neoadjuvant treatment did not significantly influence claudin 18.2 expression $(\mathrm{P}=0.331)$. Interestingly, the analyses revealed significantly decreased claudin 18.2 expression in human epidermal growth factor receptor 2 (HER2) positive tumors $(\mathrm{P}=0.036$; Table I).

OS time. Expression of claudin 18.2 in primary tumor types or lymph node metastases was not associated with a prolonged or shortened OS time in patients with EAC. The median OS time in patients with claudin 18.2 expression was 33.1 months [95\% confidence interval (CI), 8.8-57.5 months] compared with 27.1 months (95\% CI, 21.5-32.8 months; $\mathrm{P}=0.521$ ) in patients with negative claudin 18.2 expression (Fig. 2). A number of subgroup analyses were performed in order to detect specific interactions between differences in OS time and claudin 18.2 expression. However, none of the analyzed co-variables (including T-cell inflammation, mutations in TP53 and ARID1A and HER2 expression) affected the OS time in relation to claudin 18.2 expression (data not shown).

\section{Discussion}

Personalized therapy approaches for patients with EAC are urgently required due to the poor prognosis and increasing incidence of the disease. Comparative molecular analyses of gastric adenocarcinomas have revealed important differences between gastric and esophageal adenocarcinomas, including the absence of the Epstein-Barr virus and microsatellite

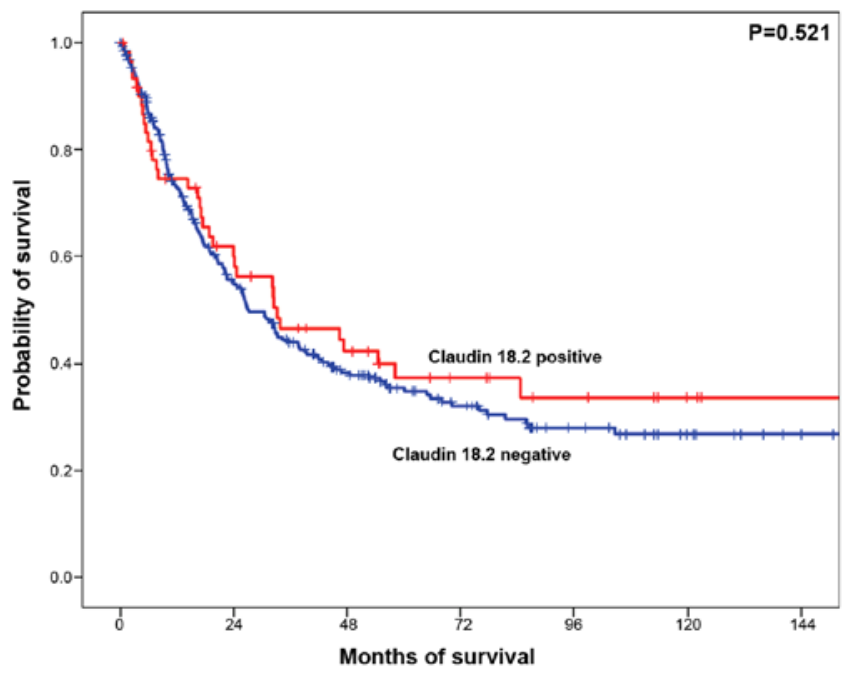

Figure 2. Median overall survival time in patients with claudin 18.2 expression compared with patients who were claudin 18.2 negative.

instability in EAC. Therefore gastric cancer expression data cannot be applied for the investigation of EAC (31-33).

Claudin 18.2 is an interesting tight-junction protein that may be therapeutically modifiable and whose relevance is currently being tested in studies on gastric cancer $(16,18-21,28)$. In these studies, the response to therapy is associated with the measurable presence of the protein in the tumor $(16,18-21,30)$. This may make claudin 18.2 a relevant biomarker, as we have known for years with programmed death-ligand 1, HER2/neu or hormone receptors in breast carcinoma. An important goal of the present study was to demonstrate how frequently claudin 18.2 expression may be expected in adenocarcinomas of the esophagus.

To best of our knowledge, the present study is the largest systematic investigation of claudin 18.2 protein expression in EAC $(n=485)$ in addition to the first study to detect claudin 18.2 expression in corresponding regional lymph node metastases using a commercially available monoclonal antibody, which was also used in other studies on gastric cancer $(18-20,30)$. The present study demonstrated detectable claudin 18.2 expression in up to $18.4 \%$ of EAC cases. The only other study that has analyzed EAC so far selected a substantially smaller number of tumor samples $(n=22)$, used a self-developed antibody, and identified an expression of claudin 18.2 in $78 \%$ of all samples (15). The different expression rates may be due to the smaller number of tissue samples analyzed and/or due to a different antibody used in this previous study (15). Additionally, the present study revealed an expression of claudin 18.2 in a significant number of the corresponding lymph node metastases, highlighting the importance of claudin 18.2 for the development of novel targeted therapies for both primary tumor types and lymph node metastases.

Since clinical and molecular data on the patient populations in the present study were available, the potential to perform statistical analyses in parallel was an option. The decisive characteristic of a therapy-relevant biomarker is ultimately its presence and the associated response to therapy. Whether it allows additional prognostic statements is at best of 
secondary academic interest but not biologically significant. For this reason, however, the present study aimed to address the academically interesting secondary question and it may be stated that there is no additional prognostic relevance of claudin 18.2. This in no way diminishes its importance as a therapeutic biomarker. Nevertheless, the present study identified a statistically measurable association between missing claudin 18.2 expression and the presence of Her2/neu expression/amplification without serious pathophysiological explanations. It may be hypothesized that an ultimately proliferation-increasing tyrosine kinase, including Her2/neu, benefits from a missing tight-junction protein, since a rapidly dividing tumor cell may be impeded in its invasiveness by an existing cell-cell contact via tight-junctions, so that an additive effect beneficial for the tumor cells is formed here.

Regarding claudin 18.2 as a potential target for immunotherapy, the present results have an impact on potential future therapeutic strategies. IMAB362, a novel antibody targeting claudin 18.2 , has been investigated in various phase I and phase II studies for patients with gastric and/or gastro-esophageal junction cancer and has exhibited anti-tumor activity $(16,18-21,28)$. Gastrointestinal toxicities were the most commonly observed treatment-associated adverse events (18-20,30).

The studies cited above exhibited good overall tolerability of IMAB362. In three of the studies listed here, the antitumor activity of IMAB362 was demonstrated in gastric adenocarcinoma and gastroesophageal transition. Serious side effects were not observed. The most frequently observed side effects, including nausea and vomiting, should be easily treatable by already well-known potent anti-emetics (18-20,30). These results provide an outlook for an effective and low-side effect therapy in patients with advanced or metastatic EAC, which should be investigated in future clinical trials.

In conclusion, targeted therapies with manageable side effects are urgently required to improve the outcome of patients with locally advanced or metastasized esophageal carcinomas. The results obtained in the present study suggested that claudin 18.2 may serve as a novel therapeutic target in EAC.

\section{Acknowledgements}

Not applicable.

\section{Funding}

Not applicable.

\section{Availability of data and materials}

The datasets used and/or analyzed in the present study are available from the corresponding author on reasonable request.

\section{Authors' contributions}

AQ designed the study. AQ and HL scored the staining intensity for the immunohistochemistry. FG, VM and AQ analyzed and interpreted the data. VM and FG wrote the manuscript. AQ, VM, EC, HL, CB, WS and RB: made substantial contributions to conception and design and acquisition of data.FG, TZ,
AT and HA: made substantial contributions to analysis and interpretation of data. All authors reviewed the final manuscript for publication.

\section{Ethics approval and consent to participate}

Procedures were followed as outlined in accordance with ethical standards formulated in the Helsinki Declaration 1995 (and revised in 2000). Written informed consent was obtained from all patients for the usage of their tumor specimens; and ethical approval was obtained from the University of Cologne Ethics Committee (reference number: 13-091).

\section{Patient consent for publication}

Not applicable.

\section{Competing interests}

The authors declare that they have no competing interests.

\section{References}

1. Global Burden of Disease Cancer Collaboration, Fitzmaurice $C$ Akinyemiju TF, Al Lami FH, Alam T, Alizadeh-Navaei R, Allen C, Alsharif U, Alvis-Guzman N, Amini E, et al: Global, regional, and national cancer incidence, mortality, years of life lost, years lived with disability, and disability-adjusted life-years for 29 cancer groups, 1990 to 2016: A systematic analysis for the global burden of disease study. JAMA Oncol 1: 1533-1568, 2018.

2. Lepage C, Rachet B, Jooste V, Faivre J and Coleman MP: Continuing rapid increase in esophageal adenocarcinoma in England and Wales. Am J Gastroenterol 103: 2694-2649, 2008.

3. Edgren G, Adami HO, Weiderpass E and Nyren O: A global assessment of the oesophageal adenocarcinoma epidemic. Gut 62: 1406-1414, 2013.

4. Al-Batran SE, Goetze TO, Mueller DW, Vogel A, Winkler M, Lorenzen S, Novotny A, Pauligk C, Homann N, Jungbluth T, et al: The RENAISSANCE (AIO-FLOT5) trial: Effect of chemotherapy alone vs. Chemotherapy followed by surgical resection on survival and quality of life in patients with limited-metastatic adenocarcinoma of the stomach or esophagogastric junction-a phase III trial of the German AIO/CAO-V/CAOGI. BMC Cancer 17: 893, 2017.

5. Pohl H, Sirovich B and Welch HG: Esophageal adenocarcinoma incidence: Are we reaching the peak? Cancer Epidemiol Biomarkers Prev 19: 1468-1470, 2010.

6. Barrett JC: Mechanisms of multistep carcinogenesis and carcinogen risk assessment. Environ Health Perspect 100: 9-20, 1993.

7. Nones K, Waddell N, Wayte N, Patch AM, Bailey P, Newell F, Holmes O, Fink JL, Quinn MCJ, Tang YH, et al: Genomic catastrophes frequently arise in esophageal adenocarcinoma and drive tumorigenesis. Nat Commun 5: 5224, 2014.

8. Li X, Galipeau PC, Paulson TG, Sanchez CA, Arnaudo J, Liu K, Sather CL, Kostadinov RL, Odze RD, Kuhner MK, et al: Temporal and spatial evolution of somatic chromosomal alterations: A case-cohort study of barrett's esophagus. Cancer Prev Res (Phila) 7: 114-127, 2014.

9. Robert Koch-Institut (Hrsg) und die Gesellschaft der epidemiologischen Krebsregister in Deutschland e.V. (Hrsg). Berlin. Krebs in Deutschland für 2013/2014. 2017.

10. Tustumi F, Kimura CM, Takeda FR, Uema RH, Salum RA, Ribeiro-Junior U and Cecconello I: Prognostic factors and survival analysis in esophageal carcinoma. Arq Bras Cir Dig 29: 138-141, 2016.

11. Tsukita S, Furuse $M$ and Itoh M: Multifunctional strands in tight junctions. Nat Rev Mol Cell Biol 2: 285-293, 2001.

12. Tsukita S, Yamazaki Y, Katsuno T, Tamura A and Tsukita S: Tight junction-based epithelial microenvironment and cell proliferation. Oncogene 27: 6930-6938, 2008.

13. Van Itallie CM and Anderson JM: Claudins and epithelial paracellular transport. Annu Rev Physiol 68: 403-429, 2006 
14. Mineta K, Yamamoto Y, Yamazaki Y, Tanaka H, Tada Y, Saito K, Tamura A, Igarashi M, Endo T, Takeuchi K and Tsukita S: Predicted expansion of the claudin multigene family. FEBS Lett 585: 606-612, 2011.

15. Sahin U, Koslowski M, Dhaene K, Usener D, Brandenburg G, Seitz G, Huber C and Türeci O: Claudin-18 splice variant 2 is a pan-cancer target suitable for therapeutic antibody development. Clin Cancer Res 14: 7624-7634, 2008.

16. Singh P, Toom S and Huang Y: Anti-Claudin 18.2 antibody as new targeted therapy for advanced gastric cancer. J Hematol Oncol 10: 105, 2017.

17. Woll S, Schlitter AM, Dhaene K, Roller M, Esposito I, Sahin U, and Türeci Ö: Claudin 18.2 is a target for IMAB362 antibody in pancreatic neoplasms. Int J Cancer 134: 731-739, 2014.

18. Sahin U, Schuler M, Richly H, Bauer S, Krilova A, Dechow T, Jerling M, Utsch M, Rohde C, Dhaene K, et al: A phase I dose-escalation study of IMAB362 (Zolbetuximab) in patients with advanced gastric and gastro-oesophageal junction cancer. Eur J Cancer 100: 17-26, 2018.

19. Al-Batran S, Al-Batran SE, Schuler MH, et al: FAST: An international, multicenter, randomized, phase II trial of epirubicin, oxaliplatin, and capecitabine (EOX) with or without IMAB362, a first-in-class anti-CLDN18.2 antibody, as first-line therapy in patients with advanced CLDN18.2+ gastric and gastroesophageal junction (GEJ) adenocarcinoma. J Clin Oncol 34 (no. 18-Suppl), 2016.

20. Trarbach T, Schuler M, Zvirbule Z, Lordick F, Krilova A, Helbig U, Schulze-Bergkamen H, Thuss-Patience PC, Wichert G, Schmiegel W, et al: 636P-Efficacy and safety of multiple doses of IMAB362 in patients with advanced gastro-esophageal cancer: Results of a phase II study. Ann Oncol 25 (Suppl 4): iv210-iv253, 2014

21. Sahin U, Al-Batran SE, Hozaeel W, et al: IMAB362 plus zoledronic acid (ZA) and interleukin-2 (IL-2) in patients (pts) with advanced gastroesophageal cancer (GEC): Clinical activity and safety data from the PILOT phase I trial. J Clin Oncol 33 , (no. 15_Suppl), 2015.

22. Holscher AH, Schneider PM, Gutschow C and Schroder W: Laparoscopic ischemic conditioning of the stomach for esophageal replacement. Ann Surg 245: 241-246, 2007.

23. Shapiro J, van Lanschot JJB, Hulshof MCCM, van Hagen $P$, van Berge Henegouwen MI, Wijnhoven BPL, van Laarhoven HWM, Nieuwenhuijzen GAP, Hospers GAP, Bonenkamp JJ, et al: Neoadjuvant chemoradiotherapy plus surgery versus surgery alone for oesophageal or junctional cancer (CROSS): Long-term results of a randomised controlled trial. Lancet Oncol 16: 1090-1098, 2015.
24. Hoeppner J,Lordick F, Brunner T, Glatz T, Bronsert P, Rothling N, Schmoor C, Lorenz D, Ell C, Hopt UT and Siewert JR: ESOPEC: Prospective randomized controlled multicenter phase III trial comparing perioperative chemotherapy (FLOT protocol) to neoadjuvant chemoradiation (CROSS protocol) in patients with adenocarcinoma of the esophagus (NCT02509286). BMC Cancer 16: 503, 2016.

25. Wittekind $\mathrm{C}$ and Oberschmid B: Pathology and new UICC classification of esophageal carcinoma. Onkologe 16: 453-461, 2010

26. UICC. TNM: Klassifikation maligner Tumoren. 8th ed: Wiley; 2017.

27. Schneider PM, Metzger R, Schaefer H, Baumgarten F, Vallbohmer D, Brabender J, Wolfgarten E, Bollschweiler E, Baldus SE, Dienes HP and Hoelscher AH: Response evaluation by endoscopy, rebiopsy, and endoscopic ultrasound does not accurately predict histopathologic regression after neoadjuvant chemoradiation for esophageal cancer. Ann Surg 248: 902-908, 2008.

28. Simon R, Mirlacher M and Sauter G: Tissue microarrays. Method Mol Med 114: 257-268, 2005.

29. Helbig D, Ihle MA, Putz K, Tantcheva-Poor I, Mauch C, Buttner $R$ and Quaas A: Oncogene and therapeutic target analyses in atypical fibroxanthomas and pleomorphic dermal sarcomas. Oncotarget 7: 21763-21774, 2016.

30. Tureci O, Sahin U, Schulze-Bergkamen H, Zvirbule Z, Lordick F, Koeberle D, Thuss-Patience P, Ettrich T, Arnold D, Bassermann F, et al: A multicentre, phase IIa study of zolbetuximab as a single agent in patients with recurrent or refractory advanced adenocarcinoma of the stomach or lower oesophagus: The MONO study. Ann Oncol 30: 1487-1495, 2019.

31. Fukayama $M$ and Ushiku T: Epstein-Barr virus-associated gastric carcinoma. Pathol Res Pract 207: 529-537, 2011.

32. Cancer Genome Atlas Research Network: Comprehensive molecular characterization of gastric adenocarcinoma. Nature 513: 202-209, 2014

33. Hewitt LC, Inam IZ, Saito Y, Yoshikawa T, Quaas A, Hoelscher A, Bollschweiler E, Fazzi GE, Melotte V, Langley RE, et al: Epstein-Barr virus and mismatch repair deficiency status differ between oesophageal and gastric cancer: A large multi-centre study. Eur J Cancer 94: 104-114, 2018.

This work is licensed under a Creative Commons Attribution-NonCommercial-NoDerivatives 4.0 International (CC BY-NC-ND 4.0) License. 